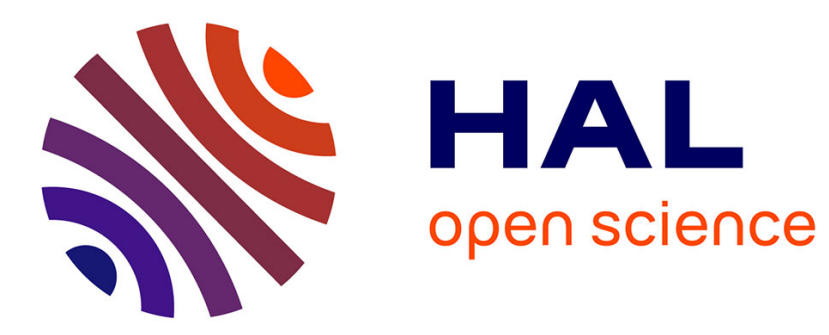

\title{
Mesure de la densité des ions métastables d'argon dans un plasma fortement ionisé
}

\author{
J. Jolly
}

\section{To cite this version:}

J. Jolly. Mesure de la densité des ions métastables d'argon dans un plasma fortement ionisé. Journal de Physique Lettres, 1975, 36 (12), pp.297-300. 10.1051/jphyslet:019750036012029700 . jpa-00231213

\section{HAL Id: jpa-00231213 https://hal.science/jpa-00231213}

Submitted on 1 Jan 1975

HAL is a multi-disciplinary open access archive for the deposit and dissemination of scientific research documents, whether they are published or not. The documents may come from teaching and research institutions in France or abroad, or from public or private research centers.
L'archive ouverte pluridisciplinaire HAL, est destinée au dépôt et à la diffusion de documents scientifiques de niveau recherche, publiés ou non, émanant des établissements d'enseignement et de recherche français ou étrangers, des laboratoires publics ou privés. 


\title{
MESURE DE LA DENSITÉ DES IONS MÉTASTABLES D’ARGON DANS UN PLASMA FORTEMENT IONISÉ
}

\author{
J. JOLLY \\ Laboratoire de Physique des Plasmas (*), Université Paris-Sud, 91405 Orsay, France \\ (Reçu le 18 septembre 1975, révisé le 30 septembre 1975, accepté le 3 octobre 1975)
}

\begin{abstract}
Résumé. - On mesure la densité des ions métastables d'argon créés dans une décharge à forte intensité de courant en utilisant une méthode d'autoabsorption. Dans la gamme de 170 à $1000 \mathrm{~A} / \mathrm{cm}^{2}$, la population des niveaux métastables $3 \mathrm{~d}^{4} \mathrm{~F}_{7 / 2}$ et $3 \mathrm{~d}^{4} \mathrm{D}_{7 / 2}$ varie linéairement en fonction de la densité électronique ainsi que le confirme l'étude des mécanismes de création et de destruction des ions métastables.
\end{abstract}

\begin{abstract}
The measurement of the concentration of metastable ions in a high-current-density argon discharge is made by self-absorption technique. For current densities ranging from 170 to $1000 \mathrm{~A} / \mathrm{cm}^{2}$, the populations of $3 \mathrm{~d}^{4} \mathrm{D}_{7 / 2}$ and $3 \mathrm{~d}^{4} \mathrm{~F}_{7 / 2}$ levels are found to be linear functions of electron density as predicted by the study of excitation and de-excitation mechanisms.
\end{abstract}

1. Introduction. - Les ions métastables des gaz rares jouent un rôle important dans divers processus d'excitation ou de transfert de charge en physique atomique et moléculaire. Dans les lasers ioniques ils contribuent, comme niveau intermédiaire, au peuplement par collision électronique du niveau laser supérieur [1]. Les ions métastables sont également utilisés comme source d'énergie dans les expériences de post-décharge pour produire, par transfert de charge, des ions moléculaires excités [2]. Par exemple pour former, dans l'azote, l'état $\mathrm{N}_{2}^{+}\left(\mathrm{B}^{2} \Sigma_{\mathrm{u}}^{+}\right)$avec des ions $\mathrm{Ar}^{+}, \mathrm{Kr}^{+}$ou $\mathrm{Xe}^{+}$, ce sont les niveaux métastables qui interviennent puisque les ions dans l'état fondamental ne sont pas capables de produire ces réactions à température ambiante [3].

Si l'importance des ions métastables a été constatée par de nombreux travaux, peu d'études ont été consacrées à la mesure de leur densité de population. Nous présentons ici les mesures des populations de 2 niveaux métastables de l'ion $\mathrm{Ar}^{+}$obtenus dans un plasma fortement ionisé créé par une décharge électrique en régime pulsé.

2. Dispositif expérimental et méthode de mesure. Le plasma est obtenu dans une décharge électrique fonctionnant en régime pulsé pour des pressions d'argon comprises entre $2 \times 10^{-2}$ et $10^{-1}$ torr. Les impulsions de courant de forme carrée durent $25 \mu$ s et leur intensité peut être réglée entre 50 et

(*) Laboratoire associé au C. N. R. S.
$300 \mathrm{~A}\left(175 \mathrm{à} 1050 \mathrm{~A} / \mathrm{cm}^{2}\right.$ dans un tube de diamètre $6 \mathrm{~mm})$.

Les techniques d'absorption habituellement employées pour mesurer les densités d'atome métastable sont difficilement applicables aux ions métastables obtenus dans ce type de décharge. Nous avons utilisé une méthode d'autoabsorption permettant de déterminer le coefficient d'absorption du milieu à partir de la mesure du rapport des intensités totales de deux raies partiellement autoabsorbées aboutissant sur le niveau dont on veut déterminer la population. Cette méthode, développée pour un milieu dans lequel les raies présentent un profil de Gauss, de Lorentz ou de Voigt, a été présentée dans une précédente publication [4].

On rappelle rapidement le principe de la méthode dans le cas, étudié ici, où l'effet Doppler est prédominant. On considère deux transitions optiques de longueur d'onde $\lambda_{1}$ et $\lambda_{2}$ aboutissant sur le niveau dont on veut déterminer la population, $f_{1}$ et $f_{2}$ sont les forces d'oscillateur correspondantes avec $f_{1}>f_{2}$. On mesure le rapport des intensités totales des raies 1 et 2 émises dans deux directions correspondant à des longueurs d'absorption différentes $l_{x}$ et $l_{y}$ avec $l_{x}>l_{y}$. Dans le cas d'une décharge dans un tube, on mesure $\left(I_{1} / I_{2}\right)_{x}$ dans l'axe du tube et $\left(I_{1} / I_{2}\right)_{y}$ transversalement au tube. Le rapport $\left(I_{1} / I_{2}\right)_{x} /\left(I_{1} / I_{2}\right)_{y}$ s'exprime en fonction du coefficient d'absorption $k_{01}$ au centre de la raie 1 , sous la forme :

$$
\left(\frac{I_{1}}{I_{2}}\right)_{x} /\left(\frac{I_{1}}{I_{2}}\right)_{y}=R \quad k_{01} l_{x}, \frac{k_{01}}{k_{02}}, \frac{l_{x}}{l_{y}},
$$


où $k_{01} / k_{02}=\left(\lambda_{1} f_{1} / \lambda_{2} f_{2}\right)$. Nous avons calculé numériquement la fonction $R\left(k_{01} l_{x}\right)$, référence [4], pour différentes valeurs des paramètres $k_{01} / k_{02}$ et $l_{x} / l_{y}$. Le coefficient d'absorption s'exprime en fonction de la densité d'ion absorbant $N\left(\mathrm{~cm}^{-3}\right)$ :

$$
k_{01}=\frac{2 e^{2}}{m c}(\pi \operatorname{Ln} 2)^{1 / 2} \frac{N f_{1}}{\Delta v_{1 \mathrm{D}}},
$$

$e$ et $m$ sont respectivement la charge et la masse de l'électron, $c$ la vitesse de la lumière et $\Delta v_{1 \mathrm{D}}$ la largeur à mi-hauteur du profil Doppler de la raie 1 , donné par :

$$
\Delta v_{1 \mathrm{D}}=2(2 R \operatorname{Ln} 2)^{1 / 2} \frac{1}{\lambda_{1}}\left(\frac{T}{M}\right)^{1 / 2},
$$

où $T$ est la température ionique du gaz, $M$ la masse molaire et $R$ la constante des gaz parfaits.

Selon l'axe de la décharge on peut supposer le plasma homogène mais radialement la distribution des populations des niveaux émetteurs peut être différente de celle des niveaux absorbants. Nous avons montré dans ce cas [4], que l'intensité transmise diffère très peu de celle d'un milieu homogène tant que le paramètre d'absorption reste faible $\left(k_{01} l_{y} \leqslant 2\right)$. On définit alors une profondeur optique équivalente,

$$
k_{0} l_{\text {eq }}=\int k_{0}(y) \mathrm{d} y \text {. }
$$

Les mesures spectroscopiques effectuées sur le plasma sont faites au moyen d'un spectromètre à la sortie duquel est placé un photomultiplicateur. Le signal du P.M. est envoyé sur un échantillonneur moyenneur utilisant des échantillons de durée $0,5 \mu \mathrm{s}$ prélevés $15 \mu \mathrm{s}$ après le départ de l'impulsion et moyennés sur un grand nombre d'impulsions. L'ensemble du dispositif expérimental est schématisé sur la figure 1.

3. Résultats expérimentaux. - Il est nécessaire de connaître les variations de la densité électronique en fonction du courant de décharge pour pouvoir interpréter les résultats. Nous avons calculé $N_{\mathrm{e}}$ à partir de la mesure de l'élargissement par effet Stark de la raie $\mathrm{H}_{\beta}$. La pression d'hydrogène ajoutée dans la décharge est de $1 \%$ de la pression d'argon. La largeur à mi-hauteur de la raie $\mathrm{H}_{\beta}$ est mesurée au moyen d'un monochromateur à haute résolution $(R>30000)$. La largeur observée est celle d'un profil de Voigt résultant de la superposition d'un profil de Gauss et d'un profil de Lorentz. La largeur à mi-hauteur $\Delta \lambda_{\mathrm{L}}$ due à l'effet Stark est extraite de la largeur observée au moyen des tables de Davies et Vaughan [5]. La détermination de la densité électronique à partir de $\Delta \lambda_{\mathrm{L}}$ se fait au moyen de la formule de Griem [6] :

$$
N_{\mathrm{e}}=C\left(N_{\mathrm{e}}, T_{\mathrm{e}}\right)\left(\Delta \lambda_{\mathrm{L}}\right)^{3 / 2} .
$$

$C\left(N_{\mathrm{e}}, T_{\mathrm{e}}\right)$ est un coefficient variant rapidement en fonction de $N_{\mathrm{e}}$ et très lentement en fonction de $T_{\mathrm{e}}$ [7].

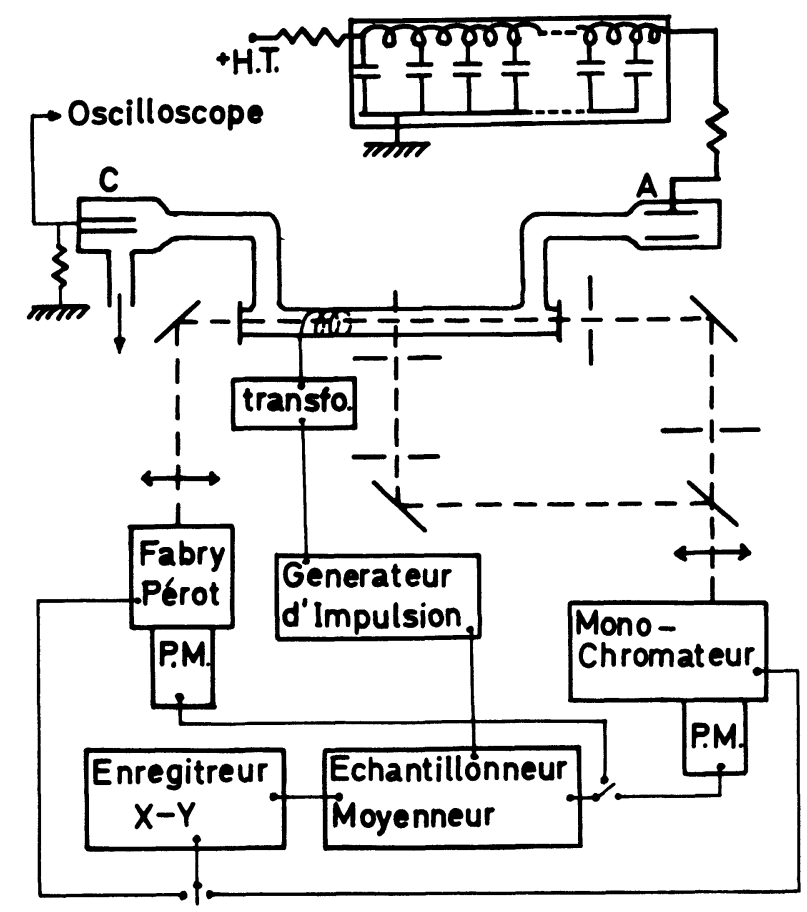

FIG. 1. - Représentation schématique du dispositif expérimental.

Les calculs ont été faits pour une température électronique de $40000 \mathrm{~K}$. Cette valeur est déterminée, dans l'hypothèse d'un milieu fortement ionisé, à partir de la mesure de la conductivité du plasma. Les résultats obtenus sont présentés figure 2. Pour les faibles courants $N_{\mathrm{e}}$ varie linéairement en fonction de l'intensité du courant de décharge. Pour les courants plus élevés une saturation apparaît probablement dûe à la raréfaction du gaz neutre.

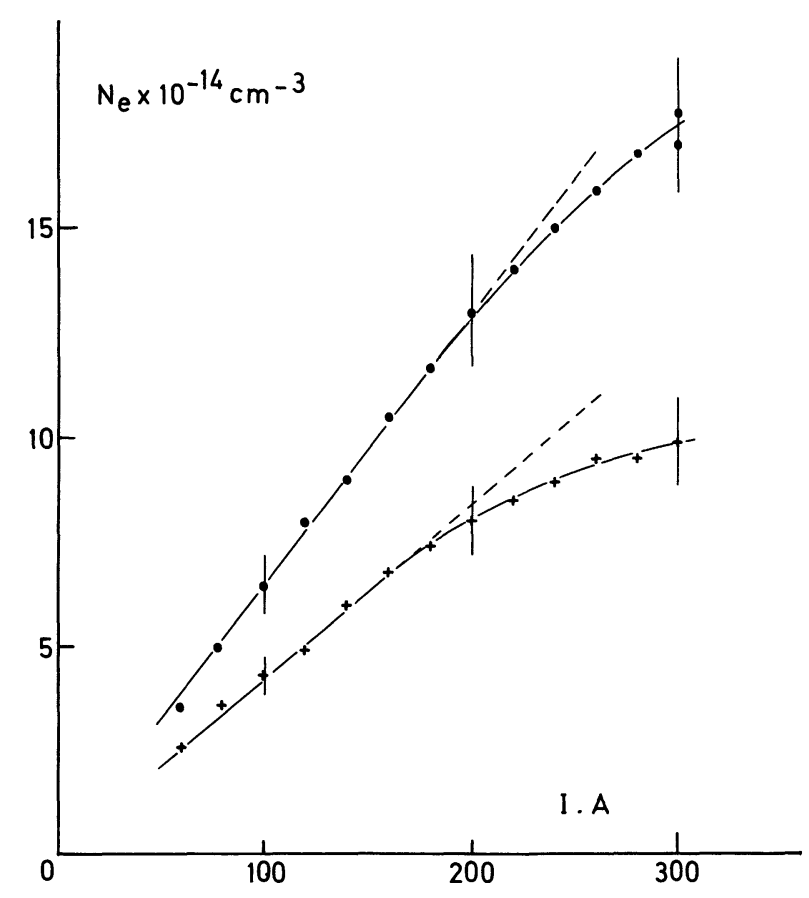

FIG. 2. - Variation de la densité électronique en fonction du courant de décharge. Pression de remplissage d'argon : $(+)$ $5 \times 10^{-2}$ torr, (•) $10^{-1}$ torr. 
Pour calculer la densité des ions métastables, d'après la formule (2), il faut connaître la largeur Doppler $\Delta v_{\mathrm{D}}$ de la transition étudiée. Nous avons tracé le profil de la raie avec un interféromètre FabryPérot dont le réglage et le balayage se font au moyen de céramiques piézoélectriques. Les mesures sont faites sur la raie $\lambda=4880 \AA\left(4 \mathrm{p}^{2} \mathrm{D}_{5 / 2}^{0} \rightarrow 4 \mathrm{~s}^{2} \mathrm{P}_{3 / 2}\right)$ isolée par un filtre interférentiel de bande passante $\Delta \lambda=10 \AA$. La largeur Doppler à mi-hauteur est extraite du profil mesuré en utilisant les courbes de déconvolution calculées par Ballik [8]. Les résultats présentés sous la forme de température ionique calculée d'après la formule (3) sont reproduits figure 3 en fonction du courant de décharge.

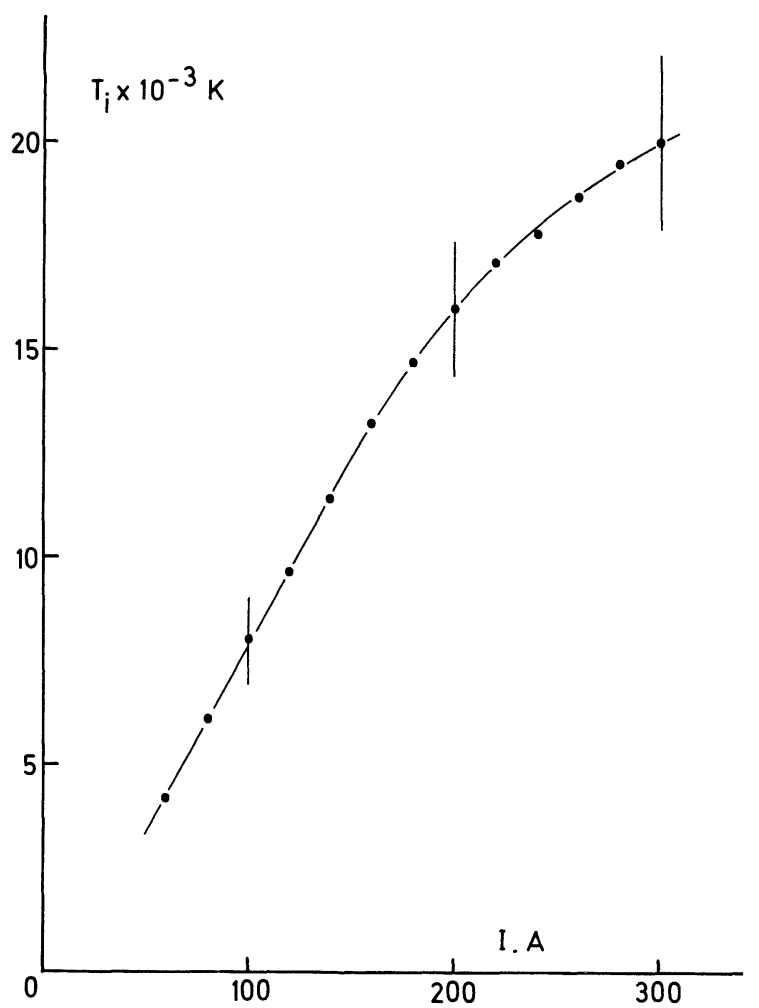

Fig. 3. - Variation de la température des ions en fonction du courant de décharge dans la gamme de pression $5 \times 10^{-2}-10^{-1}$ torr.

Nous avons mesuré la densité des ions métastables du niveau $3 \mathrm{~d}^{4} \mathrm{~F}_{7 / 2}$ et $3 \mathrm{~d}^{4} \mathrm{D}_{7 / 2}$ se trouvant respectivement à des énergies de 33,45 et 32,16 eV. Les rapports d'intensité de raies ont été mesurés en utilisant les couples de raies $6684,6243 \AA$ pour le niveau ${ }^{4} \mathrm{~F}_{7 / 2}$ et $4401,4014 \AA$ pour le niveau ${ }^{4} \mathrm{D}_{7 / 2}$. La densité des métastables ioniques s'obtient à partir du coefficient d'absorption, d'après les formules (2) et (3). On a pour l'argon,

$$
N_{\mathrm{M}}=1,375 \times 10^{5} \frac{k_{01}}{f_{1} \lambda_{1}}\left(T_{i}\right)^{1 / 2} .
$$

Les valeurs des forces d'oscillateur utilisées proviennent de Atomic Transition Probabilities [9]. Les résultats présentés sur la figure 4 correspondent aux

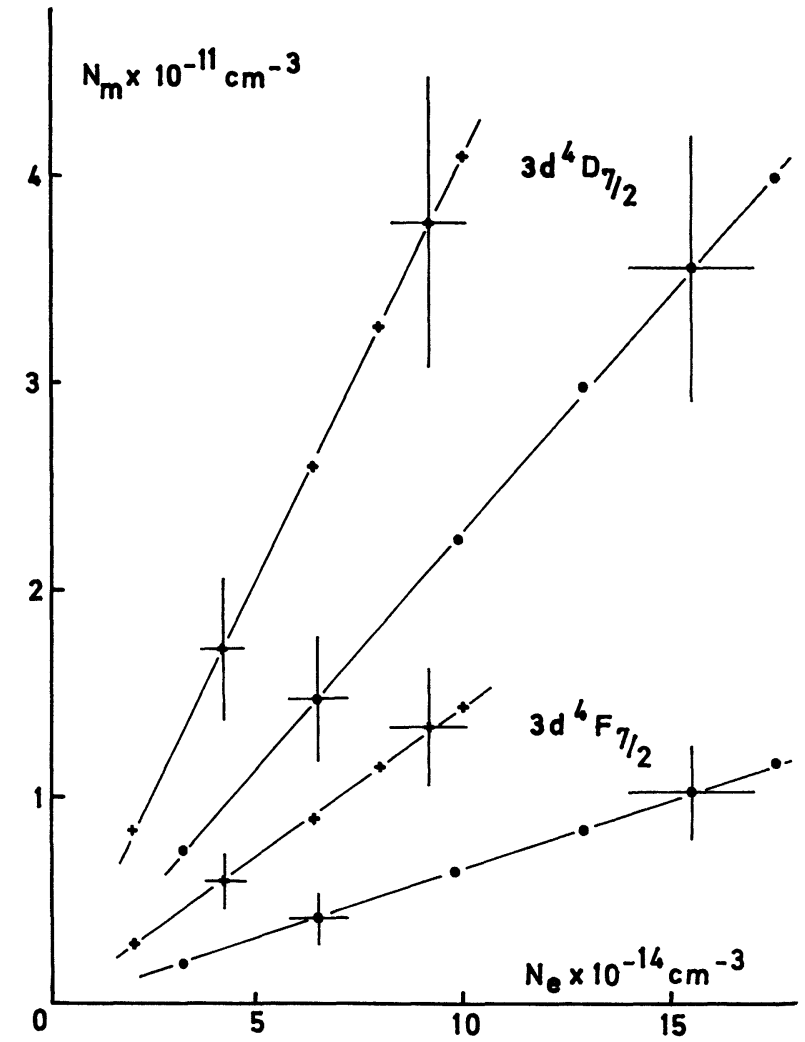

Fig. 4. - Densité des métastables ioniques pour les niveaux $3 \mathrm{~d}^{4} \mathrm{D}_{7 / 2}$ et $3 d^{4} F_{7 / 2}$ en fonction de la densité électronique. Pression de remplissage d'argon : (+) $5 \times 10^{-2}$ torr, (•) $10^{-1}$ torr.

moyennes obtenues pour trois longueurs d'absorption 8,20 et $40 \mathrm{~cm}$.

L'incertitude sur la valeur de $N_{\mathrm{M}}$ provient principalement des erreurs possibles sur la détermination de $k_{01}$ que nous évaluons à $20 \%$ ainsi que de la précision avec laquelle on connaît $f_{1}$ (la référence [9] donne pour les valeurs des forces d'oscillateur utilisées ici une précision de $25 \%$ ).

Nous présentons figure 4 les résultats obtenus pour la densité des ions métastables en fonction de la densité électronique pour des pressions de remplissage d'argon de $5 \times 10^{-2}$ et $10^{-1}$ torr.

On observe une variation linéaire de $N_{\mathrm{M}}$ en fonction de $N_{\mathrm{e}}$ dans toute la gamme de courant utilisé dans cette étude.

4. Interprétation. Mécanismes de création et de destruction des ions métastables. - Dans nos conditions expérimentales, la formation des ions excités $\mathrm{Ar}^{+*}$ se fait principalement par collision des électrons sur les ions dans leur état fondamental [1, 10]. L'évolution de la densité d'un niveau métastable ionique s'écrit :

$$
\begin{aligned}
\frac{\mathrm{d} N_{\mathrm{M}}}{\mathrm{d} t}= & N_{\mathrm{e}} N_{\mathrm{i}} C_{\mathrm{i}}^{\mathrm{M}}+ \\
& +\sum_{j>\mathrm{M}} N_{j} A_{j \mathrm{M}}-N_{\mathrm{M}} N_{\mathrm{e}} \sum_{k} C_{\mathrm{M}}^{k}-N_{\mathrm{M}} v_{\mathrm{M}} .
\end{aligned}
$$

Le premier terme du second membre correspond à l'excitation par collisions entre les électrons (densité 
$N_{\mathrm{e}}$ ) et les ions dans l'état fondamental (densité $N_{i}$ ). Le coefficient de collision $C_{\mathrm{i}}^{\mathrm{M}}$ représente le produit de la vitesse des électrons par la section efficace de collision, moyenné sur la fonction de distribution des vitesses électroniques. Le second terme rend compte de la population par cascade radiative à partir de niveaux ioniques d'énergie supérieure (densité $N_{j}$ ). Le troisième terme correspond au dépeuplement par collision électronique et le dernier à la perte par diffusion.

Dans les conditions de fonctionnement utilisées, les fréquences radiatives et collisionnelles sont de l'ordre de $10^{7}$ à $10^{8} \mathrm{~s}^{-1}$ [11], on peut donc écrire $\mathrm{d} N_{\mathrm{M}} / \mathrm{d} t=0$ et négliger la perte par diffusion devant le terme de perte par collision électronique. L'éq. (7) peut alors s'écrire :

$$
N_{\mathrm{M}}=\frac{N_{\mathrm{e}} N_{\mathrm{i}} C_{\mathrm{i}}^{\mathrm{M}}+\sum_{j>\mathrm{M}} N_{j} A_{j \mathrm{M}}}{N_{\mathrm{e}} \sum_{\boldsymbol{k}} C_{\mathrm{M}}^{k}} .
$$

Pour les niveaux $j$ pouvant alimenter les niveaux métastables par cascades radiatives on constate expérimentalement, en observant la lumière émise, une dépendance quadratique de leur population $N_{j}$ en fonction de la densité électronique. En écrivant $N_{j}=K_{j} N_{\mathrm{e}}^{2}$ et en faisant l'hypothèse de la neutralité du plasma $\left(N_{\mathrm{e}} \simeq N_{\mathrm{i}}\right)$, l'éq. (8) peut s'écrire sous la forme :

$$
N_{\mathrm{M}}=N_{\mathrm{e}} \frac{P_{\mathrm{M}}^{\mathrm{C}}\left(T_{\mathrm{e}}\right)}{Q_{\mathrm{M}}^{\mathrm{D}}\left(T_{\mathrm{e}}\right)}
$$

où $P_{\mathrm{M}}^{\mathrm{C}}\left(T_{\mathrm{e}}\right)$ et $Q_{\mathrm{M}}^{\mathrm{D}}\left(T_{\mathrm{e}}\right)$ sont respectivement les fréquences de création et de destruction. La relation (9) montre que la densité des ions métastables varie linéairement en fonction de la densité électronique si on admet que la température électronique varie peu avec le courant donc avec $N_{\mathrm{e}}$. Ceci confirme les résultats présentés figure 4.

Remerciements. - Nous remercions le Professeur J. L. Delcroix et A. Ricard pour leurs encouragements et leurs suggestions à la lecture du manuscrit.

\section{Bibliographie}

[1] LABUdA, E. F., Gordon, E. I., MilleR, R. C., I.E.E.E. J. Quantum Electron. QE-1 (1965) 273.

Bridges, W. B., Chester, A. N., Halsted, A. S., Parker, J. V., Proc. IEEE 59 (1971) 724.

[2] Kassal, T. T., Fishburne, E. S., J. Chem. Phys. 54 (1971) 1363.

[3] Moran, T. F., Fullerton, D. C., J. Chem. Phys. 56 (1972) 21.

[4] Jolly, J., Touzeau, M., J. Quant. Spectros. Radiat. Transfer. 15 (1975) 863

[5] Davies, J. T., Vaughan, J. M., Astrophys. J. 137 (1963) 1302.

[6] GrIem, H. R., Plasma Spectroscopy (Mc Graw-Hill Book Comp.) 1964, p. 170.
[7] Keprle, P., Griem, H. R., Phys. Rev. 173 (1968) 317.

[8] Ballik, E., Appl. Opt. 5 (1966) 170.

[9] Wiese, W. L., Smith, M. W., Miles, B. M., Atomic Transition Probabilities (NSRDS-NBS 22) Vol. 2 (1969).

[10] Gordon, E. I., Labuda, E. F., Miller, R. C., WebB, C. E., Proc. Conf. on Physics of Quant. Electr. (Mc Graw-Hill Book Comp.) 1966, p. 664.

Gordon, E. I., Labuda, E. F., Bridges, W. B., Appl. Phys. Lett. 4 (1964) 178.

[11] Kitaeva, V. F., Osipov, Yu. I., Rubin, P. L., Sobolev, N. N., I.E.E.E., J. Quantum Electron. 5 (1969) 72. 